\title{
Computer Simulations of Structure-Activity Relationships for hERG Channel Blockers
}

\author{
Lars Boukharta, ${ }^{\dagger}$ Henrik Keränen, ${ }^{\dagger}$ Anna Stary-Weinzinger, ${ }^{\ddagger}$ Göran Wallin, ${ }^{\dagger}$ Bert L. de Groot, ${ }^{\S}$ \\ and Johan Åquist ${ }^{*+}$ \\ ${ }^{\dagger}$ Department of Cell and Molecular Biology, Uppsala University, Biomedical Center, Box 596, SE-751 24 Uppsala, Sweden \\ ${ }^{\ddagger}$ Department of Pharmacology and Toxicology, University of Vienna, Althanstrasse 14, 1090 Vienna, Austria \\ ${ }^{\S}$ Computational Biomolecular Dynamics Group, Max Planck Institute for Biophysical Chemistry, Am Fassberg 11, \\ 37077 Göttingen, Germany
}

Supporting Information

ABSTRACT: The hERG potassium channel is of major pharmaceutical importance, and its blockade by various compounds, potentially causing serious cardiac side effects, is a major problem in drug development. Despite the large amounts of existing biochemical data on blockade of hERG by drugs and druglike compounds, relatively little is known regarding the structural basis of binding of blockers to the channel. Here, we have used a recently developed homology model of hERG to conduct molecular docking experiments with a series of channel blockers, followed by molecular dynamics simulations of the complexes and evaluation of binding free energies with the linear interaction energy method. The calculations yield a remarkably good agreement with experimental binding affinities and allow for a rationalization of three-dimensional structure-activity relationships in terms of a

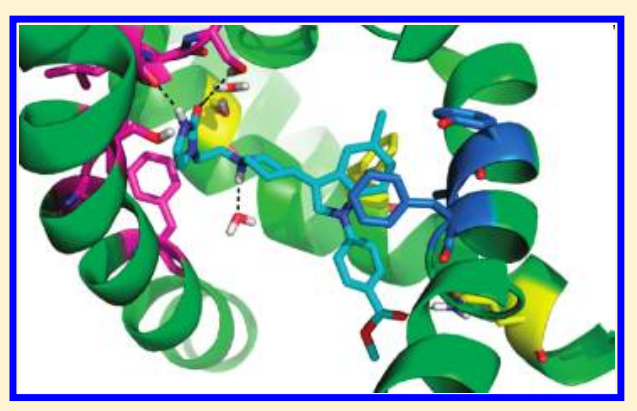
number of key interactions. Two main interaction regions of the channel are thus identified with implications for further mutagenesis experiments and design of new compounds.

$T^{1}$ he recent progress in the determination of three-dimensional (3D) structures of biological ion channels holds great promise for obtaining a structure-based quantitative view of interactions between channels and ligands of biological and pharmacological importance. Notwithstanding the increasing number of ion channel structures that have been determined, ${ }^{1-6}$ there are, however, still relatively few complexes with ligands, such as channel blockers, that have been described. ${ }^{7,8}$ From a pharmaceutical viewpoint, several of the most relevant channels have also not been structurally characterized at the atomic level. In the $\mathrm{K}^{+}$channel field, a number of key structures have been obtained $^{1-6}$ that in some cases allow for relatively reliable homology modeling of related channels and their interactions with ligands. ${ }^{1,3,5}$ Of particular interest among the human $\mathrm{K}^{+}$ channels is the human ether-à-go-go-related gene (hERG) channel that is associated with both inherited and drug-induced long QT syndrome. ${ }^{9,10}$ The latter problem, which is a side effect caused by blockade of hERG by various compounds, is a major obstacle for drug development and is presently receiving intense attention. $^{11}$

In the cardiac action potential, the hERG channel carries the rapid delayed rectifier $\left(I_{\mathrm{Kr}}\right)$ current, and its blockade leads to a prolongation of the QT interval, with severe risks for arrhythmias and sudden death. ${ }^{12,13}$ Most compounds causing such blockade apparently become trapped in the relatively unspecific internal pore cavity of the channel, and it is therefore of major interest to investigate the binding properties of this cavity. Unfortunately, the pore-forming helices of hERG show only relatively weak homology to $\mathrm{K}^{+}$channels with known 3D structure and particularly with members of the Shaker-related family. ${ }^{5,14}$ The short pore helix, the selectivity filter, and the innermost S6 helices lining the pore can, however, be confidently aligned on the basis of conservation of the filter region and a glycine hinge in S6. ${ }^{15,16}$ The situation is more ambiguous for the S5 helices that pack against the S6 helices in the tetrameric structure, and several alignments of S5 against $\mathrm{K}^{+}$channels of known structure have been published. ${ }^{15-18}$ We have recently reported an analysis of seven different alignments and 3D pore models utilizing conventional $3 \mathrm{D}$ structure quality validation methods as well as molecular dynamics (MD) simulations. From that work, one model (model 6 of ref 19) emerged as the most consistent, and it also provided a rationalization of the results from mutation scanning experiments. ${ }^{20}$

Here, we investigate the performance of our best hERG pore model with respect to prediction of binding affinities for a series of sertindole analogues listed in Table $1 .{ }^{21}$ Sertindole is an indolylpiperidine antipsychotic agent that has nanomolar affinities for dopamine $\mathrm{D}_{2}$, serotonin $5-\mathrm{HT}_{2}$, and $\alpha_{1}$ adrenergic

Received: February 3, 2011

Revised: June 9, 2011

Published: June 09, 2011 
Table 1. Sertindole Analogues Used in This Work and Their Experimental hERG IC So $_{\text {Values }}{ }^{21}$

\begin{tabular}{|c|c|c|}
\hline Compound $^{\mathrm{a}}$ & Structure & $\mathrm{hERG} \mathrm{IC}_{50}(\mathrm{nM})$ \\
\hline sertindole & & 3 \\
\hline 1 & & 88 \\
\hline 2 & & 10 \\
\hline 3 & & 7 \\
\hline 6 & & 137 \\
\hline 7 & & 131 \\
\hline 8 & & 36 \\
\hline 10 & & 6.2 \\
\hline 13 & & 23.5 \\
\hline
\end{tabular}

${ }^{a}$ The ligand numbering is adopted from ref 21 .

receptors. ${ }^{22}$ However, it also is, with an $\mathrm{IC}_{50}$ value of $3 \mathrm{nM}$, a high-affinity antagonist of $\mathrm{hERG}^{21,23}$ and is known to induce QT interval prolongation in some patients. ${ }^{24} \mathrm{With}$ regard to the issue if intake of sertindole causes an increased risk for serious arrythmatic events and sudden cardiac death, there is a difference in opinion between the Food and Drug Administration (FDA) and the manufacturer. ${ }^{25,26}$ In Europe, the marketing authorization of sertindole was suspended from 1998 to 2005, because of concerns about the increased risk of sudden death due to QT interval prolongation. As of May 2011, it is still not approved by the Food and Drug Administration for use in the United States.
The aim of this study was to be able to rationalize detailed structure-activity relationships for the series of sertindole analogues on the basis of our 3D model. Automated docking using several different scoring functions was used to find ligand starting structures for subsequent multiple MD simulations that included a realistic environment surrounding the ligands, comprising protein, ions, lipids, and water. Binding free energies were calculated with the widely used linear interaction energy (LIE) method $^{27,28}$ and contrasted with available experimental results. ${ }^{21}$ The results of the simulations are encouragingly found to be highly predictive for this series of ligands and provide a 
structure-based rationalization of the channel blocking potencies of the molecules. The origin of binding affinity differences between the compounds is further analyzed in terms of residue specific interactions that can be related to alanine scanning experiments and blockade of hERG by other types of compounds.

\section{METHODS}

Homology Modeling. The hERG homology model used in this work was termed "model 6" in our recently published analysis of structural hERG models. ${ }^{19}$ The modeling procedure, validation, and $\mathrm{MD}$ equilibration are described in detail therein. In short, Modeler $7 \mathrm{v} 7^{29}$ was used to generate a 3D model of the open conformation of hERG1. Experimental results strongly suggest that sertindole blocks the activated state of the channel. ${ }^{23}$ The crystal structure of $\mathrm{K}_{\mathrm{v}} \mathrm{AP}^{3}$ and a refined model thereof $^{30}$ were used as templates. Prior to docking, the model was embedded in an equilibrated simulation box of POPC lipids, solvated with TIP4P water, ${ }^{31}$ and equilibrated for $10 \mathrm{~ns}$. This MD simulation was performed with Gromacs version $3.3^{32}$ using the OPLS-all-atom (OPLS-AA) force field. ${ }^{33}$ The 0101(1) loading state was used for the potassium channel, meaning that a $\mathrm{K}^{+}$ion was present in the pore cavity ion binding site as well as in the second and fourth position of the selectivity filter. ${ }^{34}$ Further, $\mathrm{K}^{+}$and $\mathrm{Cl}^{-}$ions were added randomly within the solvent to neutralize the system and generate an ionic strength of $150 \mathrm{mM}$ in the MD simulations.

Docking Calculations. The studied ligands are a series of nine sertindole analogues listed in Table 1 . Their $\mathrm{IC}_{50}$ values have been reported by Pearlstein et al., ${ }^{21}$ and these nine molecules comprise all positively charged sertindole analogues with a centrally positioned basic nitrogen in that study. The central basic nitrogen in the piperidine ring of all compounds was thus protonated, which led to them all having a total charge of +1 . The protonation also gave rise to cis-trans isomerism, resulting in two diastereoisomers of each compound to consider when searching for a binding mode proposition. In sertindole, for example, the 2-(1-imidazolidin-2-one)ethyl and 5-chloro-1-(4flourophenyl)indol-3-yl substituents of the central piperidine ring are either in cis or in trans. All molecules were minimized with the OPLS-AA 2001 force field in Macromodel 9.1 (Schrödinger LCC, New York, NY) prior to docking into the pore of the equilibrated hERG homology model. Automated docking was performed using GOLD version $4.0^{35,36}$ with default genetic search parameters and 10 docking runs. Water molecules and the pore cavity $\mathrm{K}^{+}$ion were removed from the hERG model prior to docking. The binding site was defined using GOLD's flood fill option in a 12 Å radius sphere centered on a point on the symmetry axis of the channel situated approximately $6 \AA$ from the selectivity filter and in the hERG pore. A potassium ion was present in the fourth position of the selectivity filter.

In a broad search approach, the docking protocol was repeated three times, each time using a different fitness function of the three implemented in GOLD: Goldscore, ${ }^{35,36}$ Chemscore, ${ }^{37}$ and ASP. ${ }^{38}$ This resulted in a total of 30 docking solutions for each ligand isomer, distributed over several clusters of conformations. The clusters of solutions were similar for all studied analogues. Given the difficulty of this particular docking problem, sertindole was chosen as a test compound, and six docking solutions in complex with hERG were further evaluated using molecular dynamics. The six sertindole solutions were the cis and trans isomers topscored by Goldscore, Chemscore, or ASP (Figure S1 of the Supporting Information). Criteria for being considered a binding mode proposition were the structural stability during $\mathrm{MD}$, a low rmsd between the average structure of the simulation and the initial docking conformation, and, finally, a low (favorable) interaction energy between the ligand and its surroundings. This approach of discrimination and ranking of docking conformations with the LIE method has previously been successfully applied. ${ }^{39}$

Molecular Dynamics Simulations. MD simulations were performed using $\mathrm{Q}^{28}$ with the OPLS-AA force field. Force field parameters for the ligands were generated with Macromodel 9.1 (Schrödinger LCC). Simulations of the bound and free states of each ligand were conducted with spherical systems with a radius of $25 \AA$. The simulation system for the bound state was centered on a point on the symmetry axis of the channel situated approximately $6 \AA$ from the selectivity filter and in the hERG pore. The lowest-energy occupancy state (0101) of the selectivity filter was considered in the MD calculations, according to the proposed potassium channel permeation mechanism. 34,40 Ionizable residues near the edge of the spherical system were set to their neutral state, ${ }^{41}$ with the exception of the distant and solvent-exposed Glu637 amino acids that were each assigned a charge of -0.5 to attain an overall neutral and initially symmetric system seen by the ligands. ${ }^{42}$ Corrections for ligand interactions with neutralized groups or distant charged residues outside the spherical system ${ }^{41}$ were not explicitly calculated because all ligands in the data set essentially have the same positioning of their positive charge at the focus of the electric field from the pore helices. These long-range electrostatic interaction energies are instead embedded in the model as a constant contribution to the $\gamma$ term of the LIE equation given below. Each system was solvated with TIP3P water, ${ }^{31}$ the free state with a $25 \AA$ radius spherical water grid and the bound state with the water configuration from the end point of the homology model equilibration. Waters situated within $2.4 \AA$ of any solute heavy atom were removed prior to $\mathrm{MD}$.

For the solvated ion channel-ligand complexes, all atoms outside the $25 \AA$ sphere were tightly restrained to their initial coordinates and excluded from nonbonded interactions. For the free ligand in water, a weak harmonic restraint was applied to the geometrical center of the solute to keep it near the sphere center. The $\mathrm{SHAKE}^{43}$ algorithm was applied to constrain all bonds to hydrogen atoms and all solvent angles. Water molecules at the sphere surface were subjected to radial and polarization restraints according to the SCAAS model. ${ }^{28,44}$ A nonbonded interaction cutoff of $10 \AA$ was used for all atoms except for the ligand (for which no cutoff was applied), and long-range electrostatic interactions beyond the cutoff were treated with the accurate local reaction field approximation. ${ }^{45}$ The data production phase of the simulations was conducted at a constant temperature of $298 \mathrm{~K}$, and the MD time step was set to 1.5 fs. For each simulation of the ion channel-ligand complex, the system was slowly heated from 1 to $298 \mathrm{~K}$ while restraints on the solute coordinates to their initial position were gradually released. This was followed by $1.5 \mathrm{~ns}$ of unrestrained equilibration and $3 \mathrm{~ns}$ of production phase in which ligand-surrounding energies were collected every 37.5 fs. Five replicate MD simulations with different initial atomic velocities were conducted for each hERG-ligand complex, starting from the most favorable (consensus) binding mode identified below. The reported ligand-surrounding energy is taken as an average over these trajectories, and errors were estimated from the standard deviations. For each simulation of 
the free ligand in water, a 400 ps equilibration was followed by $3 \mathrm{~ns}$ of data collection and repeated five times, as described above. Further, the set of six topscoring docking solutions for sertindole were each initially evaluated by $2 \mathrm{~ns}$ production $\mathrm{MD}$ runs (Table $\mathrm{S} 1$ of the Supporting Information).

As discussed below, additional free energy perturbation (FEP) calculations were conducted to examine the binding free energy difference between compounds 10 and 3 . These closely related ligands differ only in that $\mathbf{1 0}$ has a 2 -imidazolidinethione as a headgroup instead of a 2-imidazolidinone as in the other compounds. The FEP calculations were conducted with the same system setup that was used for the regular MD simulations, and 51 FEP steps ( $\lambda$ steps) were used, with the first window corresponding to 10 and the last to 3 , with a 100 ps trajectory for each window. Five additional independent MD trajectories with the setup as described above were performed for compound 3 starting from the FEP end point.

Experimental and Computational Estimates of Binding Free Energies. Relative experimental binding free energies were estimated from $\mathrm{IC}_{50}$ values ${ }^{21}$ at $298 \mathrm{~K}$ using the common approximation $\Delta G_{\text {bind }}^{\text {obs }} \cong R T \ln \mathrm{IC}_{50}$, which can be expected to yield reliable relative free energies, whereas the absolute values are usually too positive (i.e., $\mathrm{IC}_{50}>K_{\mathrm{d}}$ ). Binding free energies were calculated from the $\mathrm{MD}$ trajectories with the linear interaction energy method. ${ }^{27}$ The method uses simulations of the ligand free in solution as well as of the protein-ligand complex to calculate the change in free energy associated with binding to the protein. In the LIE method, the difference in interaction energies between the ligand and its surroundings is used to calculate the free energy of binding via the equation

$$
\Delta G_{\mathrm{bind}}^{\mathrm{LIE}}=\alpha \Delta\left\langle U_{\mathrm{l}-\mathrm{s}}^{\mathrm{vdW}}\right\rangle+\beta \Delta\left\langle U_{\mathrm{l}-\mathrm{s}}^{\mathrm{el}}\right\rangle+\gamma
$$

where $\left\langle U_{\mathrm{l}-\mathrm{s}}^{\mathrm{vdw}}\right\rangle$ and $\left\langle U_{\mathrm{l}-\mathrm{s}}{ }^{\mathrm{e}}\right\rangle$ are MD averages of the ligandsurrounding van der Waals and electrostatic interaction energies, respectively, and $\Delta$ denotes the difference between these averages in the bound and free states. The empirical parameter $\alpha$ is a scaling coefficient for the nonpolar interactions, and in our standard parametrization of the LIE method, $\alpha=0.18{ }^{46}$ The scaling factor for the polar binding contribution for charged compounds $(\beta=0.5)$ was originally derived from the linear response approximation. ${ }^{27,46}$ Finally, $\gamma$ is a constant term obtained by regression fitting that fixes the scale for absolute binding free energies. The nature of this parameter has been related to several descriptors of the binding site, such as the hydrophobic nature of the binding site. ${ }^{47}$ This standard parametrization of the LIE method has previously been applied to many different protein-ligand complexes with good results. ${ }^{17,46-53}$

\section{RESULTS}

Docking Calculations. Of the six docked hERG-sertindole complexes initially evaluated structurally and energetically by molecular dynamics simulations, the trans sertindole diastereoisomer with a ligand starting structure predicted by Goldscore best fulfilled the considered quality criteria (Table S1 of the Supporting Information). Relative to the other complexes, this docking solution displayed both high structural stability during the course of simulation (low rmsf) and a low rmsd between the sertindole starting and MD average structure. Further, in this simulation, the ligand had the lowest interaction energy with respect to its surroundings. In the MD simulation starting from

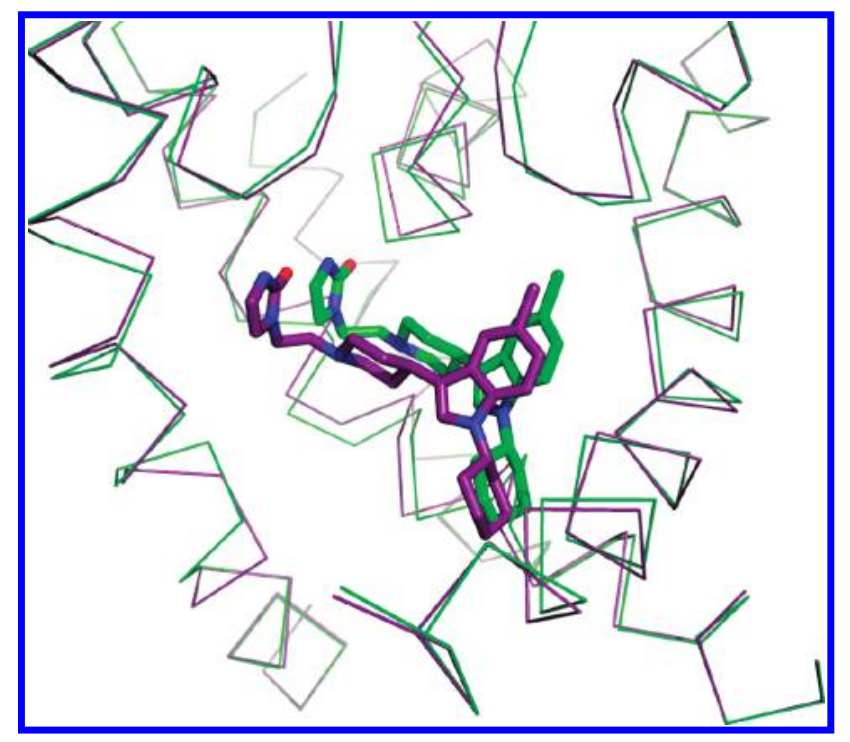

Figure 1. Initial docked structure (green) of ligand 6 compared to the average structure (purple) of a $3 \mathrm{~ns} \mathrm{MD}$ simulation.

the trans diastereomer structure predicted by Chemscore, sertindole also showed a comparably low interaction energy with its surroundings (Table S1 of the Supporting Information). However, in this simulation, the ligand partly moves out of the pore cavity and the piperidine linker and 2-imidazolidinone headgroup display considerable rotational motion within the solventfilled cavity, making this type of binding mode less well-defined. This is also reflected by higher rmsf and rmsd values versus those for the other trans solutions. Furthermore, this binding mode is not a consensus solution throughout the whole set of analogues and represents only a small fraction of the solutions for ligands 7 , 8 , and 13. The trans diastereomer was thus predicted to be in the lower-energy diequatorial form when binding, and it is worth noting that the same sertindole binding mode was also among the top-ranking solutions found by Chemscore. In fact, experimental data also have shown that the equatorial form of $\mathrm{N}$-methylpiperidine is $\sim 3 \mathrm{kcal} / \mathrm{mol}$ more stable than the axial form, ${ }^{54}$ so that our three cis-type docking solutions (with the headgroup in the axial position) could hardly represent active structures (Table S1 of the Supporting Information).

For all nine analogues, the same docking solutions were found for the trans diastereoisomer in this candidate binding mode, and these were selected as starting structures for extensive molecular dynamics and binding free energy calculations. This type of "consensus" conformation, in fact, represents $\sim 60 \%$ of all trans docking solutions. Figure 1 depicts the starting structure of ligand 6 compared to the average structure from a $3 \mathrm{~ns} \mathrm{MD}$ simulation. The average structure differs from the starting structure in that the ligand moves out slightly from the channel cavity wall because of the shift of interaction potential function from Goldscore in docking to OPLS-AA in MD. The former uses a softer $4-8$ van der Waals potential term for contacts between ligand and receptor atoms, which allows them to move closer to each other compared to a standard Lennard-Jones $6-12$ potential. $^{36}$ This choice of a softer docking potential is a common, but rather approximate, way of dealing with the issue of induced fit upon ligand binding. As a consequence, the hydrogen bond pattern of the 2-imidazolidinone moiety is shifted during MD compared to what the docking program suggests. 


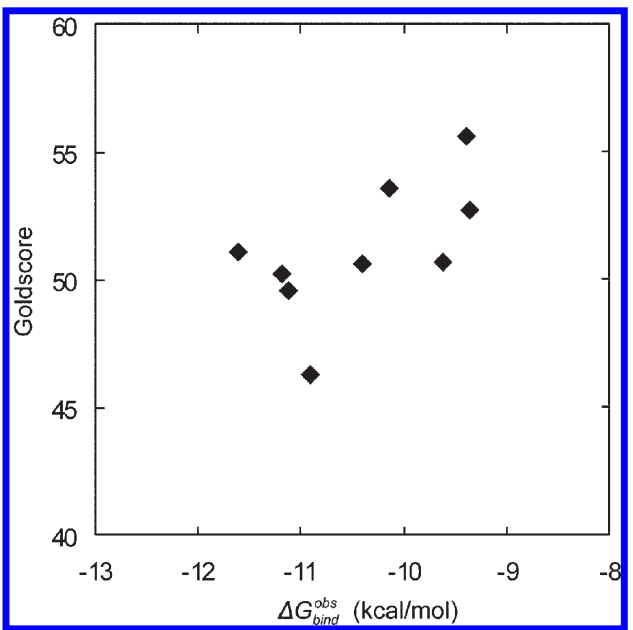

Figure 2. Goldscore vs experimental binding free energies $\left(\Delta G_{\text {bind }}^{\text {obs }}\right.$ in kilocalories per mole) for the nine hERG-ligand starting structures. Note that Goldscore is intended to be anticorrelated to binding free energy.

The Goldscore fitness function is designed to be anticorrelated to binding free energy, so good blockers are expected to have a high fitness score. In this case, the Goldscores of the candidate binding conformations instead showed a weak positive correlation $\left(r^{2}=0.37\right)$ with the experimental binding free energies (Figure 2), showing that the scoring function is not predictive for ranking of these compounds. However, it is probably not so surprising that scoring functions such as Goldscore are not able to rank these hERG-ligand complexes correctly. That is, in addition to their approximate nature, scoring functions are usually not parametrized on binding within relatively large cavities such as ion channel pores, which contain numerous water molecules and do not necessarily encapsulate blockers in a "snug fit" manner. ${ }^{7}$ Long-range electrostatic effects such as the influence of the dipole moments of the pore helices or potassium ions in the selectivity filter are not considered by these functions. The LIE method, on the other hand, takes such effects into account, and therefore, we have used it here to discriminate and rank the obtained docking conformations. ${ }^{39}$

Molecular Dynamics Simulations and LIE Binding Free Energies. Five replicate $\mathrm{MD}$ simulations with different initial atomic velocities were conducted for each hERG-ligand complex. The structures were generally stable during simulation. The rmsd values between the average structures of replicate $\mathrm{MD}$ simulations as well as between the ligand starting and average structures were low in all cases except those mentioned below. Besides visual inspection of the average $\mathrm{MD}$ structures, a principal component analysis (PCA) ${ }^{55}$ was performed in SIMCA-P 11.5 (Umetrics $\mathrm{AB}$, Umea, Sweden) to analyze the structural and energetic diversity in the five replicate $\mathrm{MD}$ runs of each ligand. Input variables for the PCA model were the electrostatic and van der Waals interaction energies among the ligands, solvent, and residues in the hERG model. Five of 45 simulations were classified as outliers because of significant structural and energetic deviation from replicate runs of the same ligand. A structural deviation was considered significant when the ligand heavy atom rmsd of one trajectory was larger than $2 \AA$ compared to both of the other replicate average structures as well as to their combined average structure. These discarded trajectories were also higher in calculated binding free energy $\left(\Delta G_{\text {bind }}^{\mathrm{LIE}}\right)$ than the replicates included in the model. An energetic deviation was considered to be significant when a single trajectory had a $\Delta G_{\text {bind }}^{\mathrm{LIE}}$ more than $1.5 \mathrm{kcal} / \mathrm{mol}$ higher than the average of all replicate simulations of the same ligand. The reported ligand-surrounding energies are taken as averages over the remaining trajectories.

The only exception to the generally stable average $\mathrm{MD}$ structures and energies was initially encountered for compound 3, which did not reach the low-energy conformation, position, and interaction pattern adopted by the other ligands sharing the same ring system, linker, and headgroup topology, when starting from the corresponding docking pose (Figure S2 of the Supporting Information). To investigate this problem, the relative binding free energy of $\mathbf{3}$ versus $\mathbf{1 0}$ was first evaluated with the FEP approach starting from the average MD structure of the complex with 10. The results from these calculations gave a relative binding free energy $\Delta \Delta G_{\mathrm{bind}^{-}}^{\mathrm{FEP}}$ $(10 \rightarrow 3)$ of $1.0 \mathrm{kcal} / \mathrm{mol}$, in reasonable agreement with the experimental value of $0.1 \mathrm{kcal} / \mathrm{mol}$ (Table 2). Five new independent $\mathrm{MD}$ simulations were therefore run, starting from the end point of the FEP calculation, and these trajectories yielded a stable, low-energy conformation with a corresponding binding free energy difference with respect to $\mathbf{1 0}$ $\left(\Delta \Delta G_{\text {bind }}^{\mathrm{LIE}}\right)$ of $0.6 \mathrm{kcal} / \mathrm{mol}$ (Table 2$)$. A comparison between the residue level ligand-surrounding interaction energies between the two sets of simulations of compound 3 shows that the energetic difference to a large extent is due to less favorable electrostatic interactions with the hERG cavity water molecules in the simulations started from the Goldscore docking pose (Figure S2 of the Supporting Information). Hence, when starting from this docking pose, the ligand became "trapped" with an unfavorable solvation pattern, which illustrates the general problem of accurately capturing important solvent molecule networks in microscopic simulations.

The resulting binding free energies calculated with the LIE method for the series of nine sertindole analogues are in excellent agreement with those derived from experimental $\mathrm{IC}_{50}$ values, as shown in Figure 3 and Table 2. The standard parametrization of the LIE method for charged compounds $(\beta=0.5$, and $\alpha=$ $0.18),{ }^{46,47}$ together with an optimized $\gamma$ of -14.1 , reproduces the experimental results with an average unsigned error of only 0.4 $\mathrm{kcal} / \mathrm{mol}\left(r^{2}=0.60\right)$. Furthermore, the ranking of the compounds is generally good, with a Spearman rank coefficient of 0.85 , and it is very encouraging that the best binders, sertindole, 2,3 , and 10 , are clearly separated from the low-affinity group consisting of $\mathbf{1 , 6}$, and 7 . It should be emphasized that the LIE model used here has no free parameters affecting relative binding free energies because the constant $\gamma$ is the same for all compounds and only sets the absolute free energy scale.

hERG - Ligand Interactions. The docked complexes in our hERG model thus show considerable stability during MD simulations, with a number of key interactions defining the consensus orientation of the ligand series (Figure 4). Hence, the 2-imidazolidinone part or headgroup of the ligands is predicted to be accommodated in a cavity formed by residues 622-624, 649, 652,653 , and 656 of the same hERG subunit. This provides polar interactions with Leu622 (backbone), Ser649, a structural water molecule, and Ser624 as well as hydrophobic interactions with Tyr652 and Phe656 (colored magenta in Figures 4 and 5). The latter three residues and Thr623 have been implicated as determinants of blocker binding in several experimental studies. ${ }^{56-62}$ The interactions with this part of the ligands also 
Table 2. Ligand-Surrounding and Calculated LIE Binding Free Energies from the MD Simulations ${ }^{a}$

\begin{tabular}{|c|c|c|c|c|c|c|}
\hline ligand & $\left\langle U_{\mathrm{l}-\mathrm{s}}^{\mathrm{vdW}}\right\rangle_{\mathrm{b}}$ & $\left\langle U_{\mathrm{l}-\mathrm{s}}^{\mathrm{vdW}}\right\rangle_{\mathrm{f}}$ & $\left\langle U_{1-\mathrm{s}}^{\mathrm{el}}\right\rangle_{\mathrm{b}}$ & $\left\langle U_{1-s}^{\mathrm{el}}\right\rangle_{\mathrm{f}}$ & $\Delta G_{\text {bind }}^{\mathrm{LIE}}$ & $\Delta G_{\text {bind }}^{\text {obs }}$ \\
\hline sertindole & $-61.5 \pm 0.4$ & $-40.2 \pm 0.1$ & $-107.3 \pm 1.0$ & $-120.9 \pm 0.4$ & $-11.1 \pm 0.5$ & -11.6 \\
\hline 1 & $-61.2 \pm 0.3$ & $-40.1 \pm 0.0$ & $-101.4 \pm 1.2$ & $-118.2 \pm 0.2$ & $-9.5 \pm 0.6$ & -9.6 \\
\hline 2 & $-63.2 \pm 0.5$ & $-40.5 \pm 0.0$ & $-101.2 \pm 2.2$ & $-114.5 \pm 0.1$ & $-11.5 \pm 1.1$ & -10.9 \\
\hline 3 & $-58.4 \pm 0.2$ & $-38.3 \pm 0.0$ & $-107.1 \pm 1.1$ & $-120.7 \pm 0.2$ & $-10.9 \pm 0.6$ & -11.1 \\
\hline 6 & $-62.9 \pm 0.3$ & $-41.3 \pm 0.0$ & $-99.3 \pm 2.0$ & $-116.5 \pm 0.5$ & $-9.4 \pm 1.0$ & -9.4 \\
\hline 7 & $-69.7 \pm 1.4$ & $-46.3 \pm 0.1$ & $-107.1 \pm 1.0$ & $-124.9 \pm 0.1$ & $-9.4 \pm 0.5$ & -9.4 \\
\hline 8 & $-67.7 \pm 0.4$ & $-45.3 \pm 0.0$ & $-108.0 \pm 1.9$ & $-122.2 \pm 0.2$ & $-11.0 \pm 0.9$ & -10.1 \\
\hline 10 & $-58.8 \pm 1.0$ & $-39.5 \pm 0.1$ & $-102.2 \pm 1.1$ & $-114.3 \pm 0.3$ & $-11.5 \pm 0.5$ & -11.2 \\
\hline 13 & $-57.6 \pm 0.4$ & $-37.4 \pm 0.1$ & $-106.4 \pm 1.9$ & $-122.7 \pm 0.4$ & $-9.6 \pm 1.0$ & -10.4 \\
\hline
\end{tabular}

${ }^{a}$ All values are in kilocalories per mole. Ligand-surrounding van der Waals $\left(\left\langle U_{\mathrm{l}-\mathrm{s}}^{\mathrm{vdW}}\right\rangle\right)$ and electrostatic $\left(\left\langle U_{\mathrm{l}-\mathrm{s}}^{\mathrm{el}}\right\rangle\right)$ energies for the bound $(\mathrm{b})$ and free $(\mathrm{f})$ states of the ligands. The energies are averages over all replicate trajectories of each ligand, and the errors correspond to the standard deviations (standard errors of the mean are lower by a factor of $\sqrt{ } 5)$. The calculated binding free energies $\left(\Delta G_{\text {bind }}^{\mathrm{LIE}}\right)$ are calculated using eq 1 with the standard parametrization of $\operatorname{LIE}^{46}$ and an optimized $\gamma$ value of -14.1 . The experimental binding free energies $\left(\Delta G_{\text {bind }}^{\text {obs }}\right)$ are derived from the relationship $\Delta G_{\text {bind }}^{\text {obs }} \cong R T \ln \mathrm{IC}_{50}$, with a $1 \mathrm{M}$ standard state.

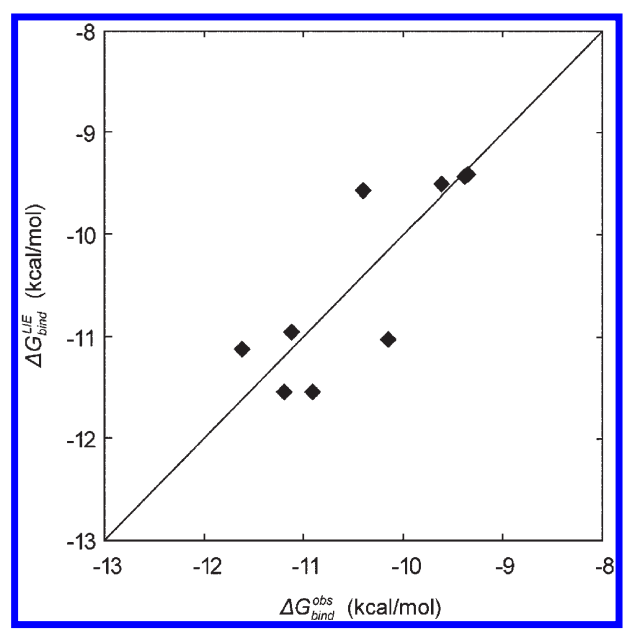

Figure 3. Calculated LIE $\left(\Delta G_{\text {bind }}^{\mathrm{LIE}}\right)$ vs experimental $\left(\Delta G_{\text {bind }}^{\text {obs }}\right)$ binding free energies (kilocalories per mole) for the nine sertindole analogues. The solid line represents perfect agreement between $\Delta G_{\text {bind }}^{\mathrm{LIE}}$ and $\Delta G_{\text {bind. }}^{\text {obs. }}$

share common features with the earlier model of Österberg et $\mathrm{al}^{17}$

The central piperidine moiety, on the other hand, forms no specific interactions with the channel but places the positively charged nitrogen near the focus of the electric field from the pore helices (as also predicted previously ${ }^{17}$ ), where cations have been observed in crystal structures. ${ }^{3,5,7,8}$ It is further interesting to note that this region of the molecules is relatively highly solvated by water molecules residing in the upper part of the central channel cavity, and the protonated nitrogen can make hydrogen bonds with the solvent (Figure 4C). The positively charged region of the ligand also has a favorable polar interaction with the side chain and backbone of a copy of Ser649 (colored yellow in Figures 4 and 5).

The indole moiety is sequestered by two copies of Phe656 and one copy of Tyr652 (colored yellow and blue in Figures 4 and 5). In the cases with chlorine substituents, these also interact with Ser649 and the backbone of residues 622 and 623, at the C-terminal end of one of the four P-helices (Figure 4A). The fluorobenzyl group of sertindole and its equivalents in the other compounds are predicted to pack against a hydrophobic part of the wall of the central cavity near Phe656. Here, some of the compounds are able to interact favorably with the side chain of Gln664 near the opening of the cavity toward the intracellular solution (Figure 4).

The three regions of the channel cavity mentioned above define the rather subtle structure-activity relationships for this ligand series. This is seen not only from structural comparisons of average MD structures (Figure 4D) but also in the analysis of average interaction energies between ligands and the channel on a residue basis (Figure 5). Already from the chemical structures of the ligands (Table 1), we can appreciate that the position corresponding to the fluorobenzyl group of sertindole appears to cause the largest variations in binding affinity. Here, we find that it is mainly the dipolar interaction (or weak hydrogen bonding) with the Gln664 side chain that controls this specificity. The interaction with the fluorine substituent appears optimal in this respect, but the ester carbonyl group of $\mathbf{8}$ can also make favorable contact with Gln664 (Figure 4A). As this part of the molecule is exposed to solvent in the pore cavity of our hERG model, there is a general propensity for rotational motion of the entire phenyl ring substituent. The main effect of Gln664 is an increased stability of the fluorobenzyl or methylbenzoate group in sertindole and compounds $2,3,8,10$, and 13 , which also facilitates hydrophobic interaction with the cavity wall mentioned above. With a methyl phenylacetate substituent as in 7 , the distance from the ester carbonyl to Gln664 becomes too large, while compounds 1 and 6 lack a polar moiety altogether, rendering these blockers less potent. It is also apparent that interaction with the cavity wall is more favorable for a phenyl than for a cyclohexanyl ring, as shown by the comparison of $\mathbf{1}$ and $\mathbf{6}$.

The indole group is tightly positioned among Tyr652, Ala653, and two copies of Phe656 (Figure 4) and is a key feature of the series. These contacts give rise to the favorable and relatively invariant nonpolar interactions between these residues and the ligands seen in Figure 5B. The chlorine substituent in sertindole has a relatively weak effect on both affinity and structure as judged from the MD simulations. This is also supported by the small reduction in affinity seen experimentally upon removal of the chlorine (as in 3). The planarity of the indole ring system is, however, important for positioning of the entire linker to the headgroup. In compound 13, which instead comprises an indoline double ring, the linker shifts its position, resulting in a $2 \AA$ 


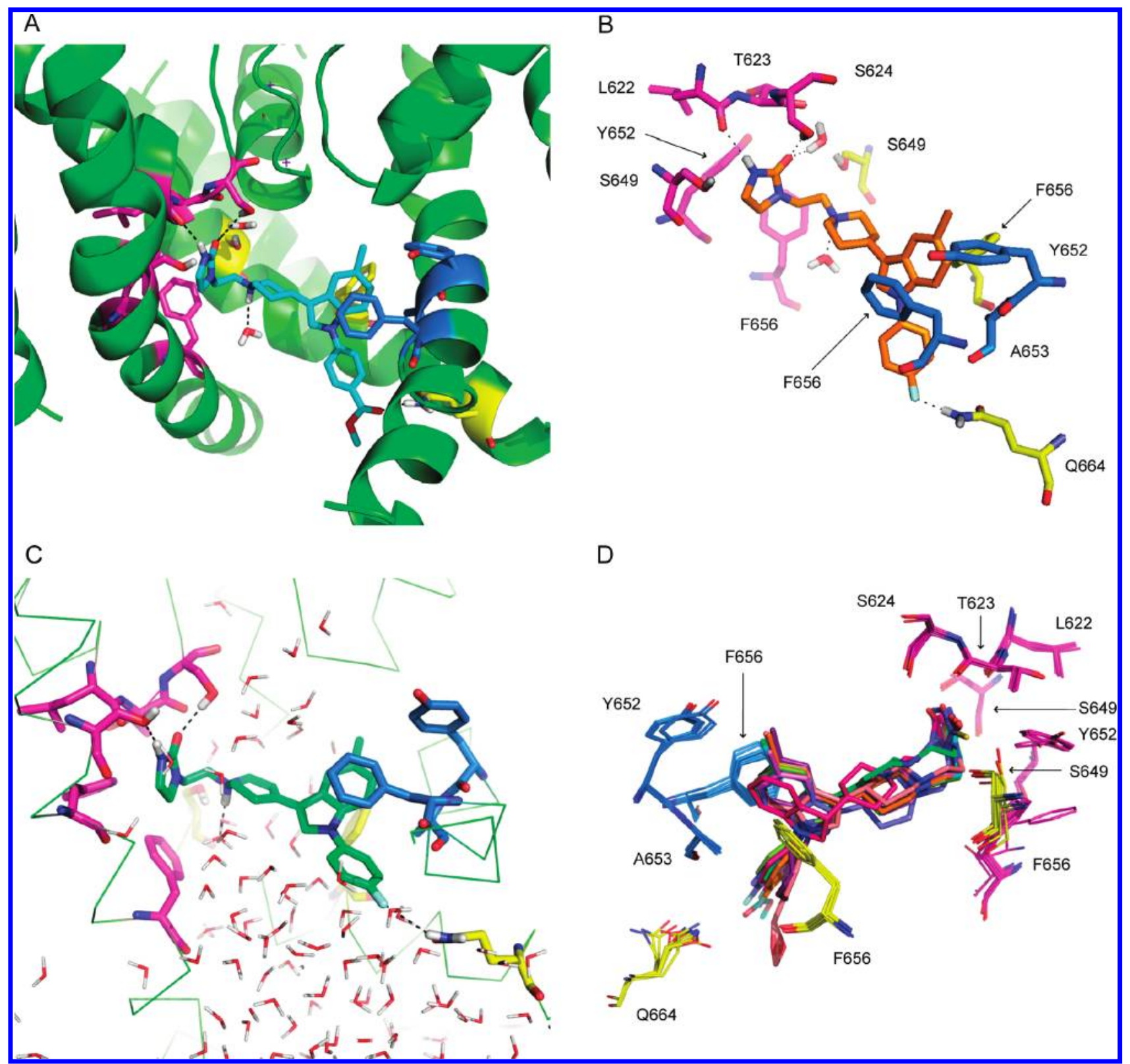

Figure 4. (A) Overview of a hERG-ligand complex showing the average MD structure of hERG (green ribbon) in complex with compound 8 (cyan sticks). Residues that contribute most to binding and/or specificity are shown in stick representation and colored magenta, yellow, or blue, depending on the hERG subunit to which they. The fourth hERG subunit that is not in direct contact with the ligands was removed for the sake of clarity. (B) Close-up of a MD average structure of sertindole (orange) and key hERG residues. Two structural water molecules and important polar interactions of sertindole are also depicted. (C) Water structure from an MD snapshot of hERG in complex with ligand $\mathbf{2}$ (green sticks). The hydrogen bond pattern of $\mathbf{2}$ is also displayed, and it differs slightly from that of sertindole. (D) Comparison of MD average structures of all nine ligand complexes. The view is from the opposite side relative to that in the other panels.

displacement of the quartenary nitrogen and also in slight but significant conformational effects on the 2-imidazolidinone headgroup (Figure 4D, ligand colored pink). This is reflected by weakened interaction with Tyr652, Phe656, and Ser649 but is partly compensated by strengthened interactions with Leu622 and Ser624, resulting in an overall affinity reduction of a factor of $\sim 10$.

The central piperidine linker ring also dictates the conformation of the ligands by its specific puckering (chair conformation). The only molecule differing in this respect is $\mathbf{2}$, which instead has a tetrahydropyridine ring. This yields a third type of positioning of the linker compared to the sertindole type and that of $\mathbf{1 3}$
(Figure 4D, ligand colored green). Nevertheless, the 2-imidazolidinone headgroup can still be accommodated in a similar manner like the other compounds but is slightly shifted and has a different hydrogen bond pattern. The hydrogen bond donated from Ser624 is more distinct and stronger for $\mathbf{2}$ than for the other ligands, and the side chain of Ser649 is the primary H-bond acceptor for the headgroup instead of the backbone carbonyl of Leu622 (Figure 4C). In this case, packing against Tyr652 and Phe656 is not affected as much as that with 13.

Finally, it is interesting to compare the differences between 2-imidazolidinone and 2-imidazolidinethione at the headgroup, 


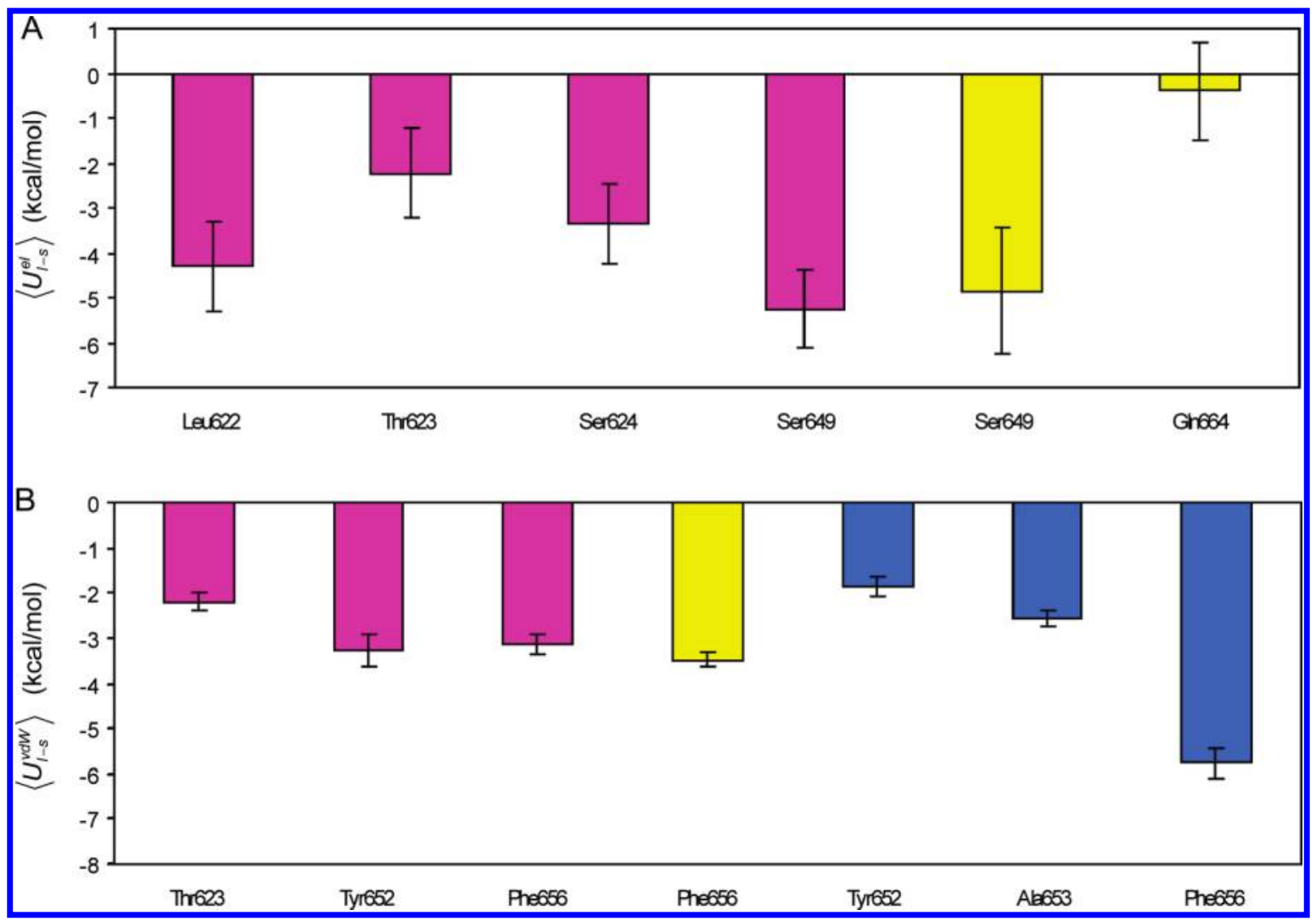

Figure 5. Average ligand-residue interaction energies $\left(\left\langle U_{\mathrm{l}-\mathrm{s}}^{\mathrm{el}}\right\rangle\right.$ and $\left.\left\langle U_{\mathrm{l}-\mathrm{s}}^{\mathrm{ddW}}\right\rangle\right)$ over all nine ligands used in the LIE calculations for the residues that contribute most to the ligand-surrounding (A) electrostatic and (B) van der Waals energies and/or the binding specificity. The average interaction energies and the corresponding standard deviations are shown using regular bars and error bars, respectively. The coloring is equivalent to that in Figure 4, with colors associated with different hERG subunits.

i.e., 10 versus 3 . The bulkier nature and reduced $\mathrm{H}$-bond capability of the sulfur atom in $\mathbf{1 0}$ also cause a shift of the headgroup that also affects the linker region (Figure 4D, ligand colored blue). However, in this case, the protonated nitrogen on the headgroup becomes well positioned for donating an $\mathrm{H}$-bond to Ser624 in contrast to the other compounds that instead accept an H-bond from this residue. The hydrophobic interaction with Phe656 is also strengthened in 10 compared to the other ligands. Apparently, the cavity that accommodates the headgroup provides several H-bond possibilities, and 2, 13, and 10 each display unique hydrogen bond patterns compared to the pattern of the other six ligands sharing both ring systems and the 2 -imidazolidinone moieties.

\section{DISCUSSION}

In this work, we used a recently developed homology model of hERG to conduct molecular docking experiments, followed by molecular dynamics simulations and binding affinity prediction by the linear interaction energy method, to examine whether this model can be used to rationalize structure-activity relationships for series of sertindole analogues as channel blockers. On the basis of the initial evaluation of six top-scoring docking solutions for sertindole itself with this strategy, the favored diastereoisomer was identified and found to give a consensus docking solution among all the ligands. The corresponding channel-blocker complexes are notably stable during $\mathrm{MD}$ simulations and the calculated binding free energies in surprisingly good agreement with experimental binding data.
The observed stability of the docked complexes is mainly due to two well-defined interaction regions of the channel, in combination with positioning of the central charged nitrogen such that it can maintain interactions with solvent molecules. This location of the positive charge in the center of the internal cavity essentially coincides with that of a potassium ion or the quaternary ammonium ion nitrogen in crystal structures. The two interaction regions emerging from these calculations give rise to rather distinct structure - activity relationships that can be interpreted in light of the available experimental data. These predicted relationships could be further used both in designing new ligands and in mutagenesis experiments.

Alanine scanning approaches have been used to identify residues important for the blockade of hERG by a variety of structurally divergent compounds, ${ }^{56-65}$ and such experiments can provide useful validations of the current methodology. ${ }^{51}$ Unfortunately, to date, no investigation of sertindole block using this approach has, however, been reported. Sertindole belongs to the highly potent class of hERG blockers featuring a linear topology with a central positive ionizable nitrogen situated in a linker connecting hydrophobic groups or aromatic rings on both sides. Interestingly, all hydrophobic central amine compounds investigated by alanine scanning ${ }^{56-62}$ show reduced affinity when Thr623, Ser624, Tyr652, or Phe656 of hERG is mutated. It is therefore noteworthy that these residues represent both of the two key binding regions identified herein. They also line the pore cavity in many other reported hERG models. $^{15,17,18,56,66}$ 
A hERG alanine scanning study of sertindole blockade would clearly be very interesting in comparison with this work. Several key mutations to include in a broader hERG mutagenesis study comprising sertindole analogues could also be proposed on the basis of our results. For example, we predict that mutation of Ser624, Ser649, or Gln664 would affect the relative binding affinities of the sertindole analogues, because the ligands differ in their interactions with these side chains (Figures 4 and 5). Furthermore, measurement of the response of potent hERG blockers to an Ala653 mutant channel (e.g., Ala653Val) would be of considerable interest because this residue is omitted from alanine scanning studies. In our model, it faces the pore cavity and stacks against both the 2-imidazolidinone and indole moieties of the ligands. This residue further appears to be important for controlling the position of the adjacent Phe656 side chain from another subunit, thereby contributing to shaping the narrowest part of the hydrophobic wall that may be an important binding determinant for several types of compounds.

\section{ASSOCIATED CONTENT}

S Supporting Information. Detailed results of evaluation of sertindole docking solutions using molecular dynamics simulations and comparison of MD starting and average structures of compound 3. This material is available free of charge via the Internet at http://pubs.acs.org.

\section{AUTHOR INFORMATION}

\section{Corresponding Author}

*Phone: +46 18471 4109. Fax: +46 18536971 . E-mail: aqvist@ xray.bmc.uu.se.

\section{Funding Sources}

This work was supported by the Swedish Research Council (VR), the EC (Grant HEALTH-F4-2007-201924, EDICT) and by the Austrian Science Fund (Grant P22395).

\section{ABBREVIATIONS}

hERG, human ether-à-go-go-related gene; MD, molecular dynamics; LIE, linear interaction energy; rmsd, root-mean-square deviation; FEP, free energy perturbation; rmsf, root-meansquare fluctuation; PCA, principal component analysis.

\section{REFERENCES}

(1) Doyle, D. A., Cabral, J. M., Pfuetzner, R. A., Kuo, A. L., Gulbis, J. M., Cohen, S. L., Chait, B. T., and MacKinnon, R. (1998) The structure of the potassium channel: Molecular basis of $\mathrm{K}^{+}$conduction and selectivity. Science 280, 69-77.

(2) Jiang, Y. X., Lee, A., Chen, J. Y., Cadene, M., Chait, B. T., and MacKinnon, R. (2002) Crystal structure and mechanism of a calciumgated potassium channel. Nature 417, 515-522.

(3) Jiang, Y. X., Lee, A., Chen, J. Y., Ruta, V., Cadene, M., Chait, B. T., and MacKinnon, R. (2003) X-ray structure of a voltage-dependent $\mathrm{K}^{+}$ channel. Nature 423, 33-41.

(4) Kuo, A. L., Gulbis, J. M., Antcliff, J. F., Rahman, T., Lowe, E. D., Zimmer, J., Cuthbertson, J., Ashcroft, F. M., Ezaki, T., and Doyle, D. A. (2003) Crystal structure of the potassium channel KirBac1.1 in the closed state. Science 300, 1922-1926.

(5) Long, S. B., Campbell, E. B., and MacKinnon, R. (2005) Crystal structure of a mammalian voltage-dependent Shaker family $\mathrm{K}^{+}$channel. Science 309, 897-903.

(6) Clayton, G. M., Altieri, S., Heginbotham, L., Unger, V. M., and Morais-Cabral, J. H. (2008) Structure of the transmembrane regions of a bacterial cyclic nucleotide-regulated channel. Proc. Natl. Acad. Sci. U.S.A. 105, 1511-1515.

(7) Zhou, M., Morais-Cabral, J. H., Mann, S., and MacKinnon, R. (2001) Potassium channel receptor site for the inactivation gate and quaternary amine inhibitors. Nature 411, 657-661.

(8) Lenaeus, M. J., Vamvouka, M., Focia, P. J., and Gross, A. (2005) Structural basis of TEA blockade in a model potassium channel. Nat. Struct. Mol. Biol. 12, 454-459.

(9) Curran, M. E., Splawski, I., Timothy, K. W., Vincent, G. M., Green, E. D., and Keating, M. T. (1995) A Molecular Basis for Cardiac Arrhythmia: HERG Mutations Cause Long QT Syndrome. Cell 80, 795-803.

(10) Haverkamp, W., Breithardt, G., Camm, A. J., Janse, M. J., Rosen, M. R., Antzelevitch, C., Escande, D., Franz, M., Malik, M., Moss, A., and Shah, R. (2000) The potential for QT prolongation and proarrhythmia by non-antiarrhythmic drugs: Clinical and regulatory implications. Report on a Policy Conference of the European Society of Cardiology. Eur. Heart J. 21, 1216-1231.

(11) Pollard, C. E., Abi Gerges, N., Bridgland-Taylor, M. H., Easter, A., Hammond, T. G., and Valentin, J.-P. (2010) An introduction to QT interval prolongation and non-clinical approaches to assessing and reducing risk. Br. J. Pharmacol. 159, 12-21.

(12) Sanguinetti, M. C., Jiang, C. G., Curran, M. E., and Keating, M. T. (1995) A Mechanistic Link between an Inherited and an Acquired Cardiac Arrhythmia: HERG Encodes the $\mathrm{I}_{\mathrm{Kr}}$ Potassium Channel. Cell 81, 299-307.

(13) Trudeau, M. C., Warmke, J. W., Ganetzky, B., and Robertson, G. A. (1995) HERG, a Human Inward Rectifier in the Voltage-Gated Channel Family. Science 269, 92-95.

(14) Keating, M. T., and Sanguinetti, M. C. (2001) Molecular and cellular mechanisms of cardiac arrhythmias. Cell 104, 569-580.

(15) Stansfeld, P. J., Gedeck, P., Gosling, M., Cox, B., Mitcheson, J. S., and Sutcliffe, M. J. (2007) Drug block of the hERG potassium channel: Insight from modeling. Proteins 68, 568-580.

(16) Tseng, G. N., Sonawane, K. D., Korolkova, Y. V., Zhang, M., Liu, J., Grishin, E. V., and Guy, H. R. (2007) Probing the outer mouth structure of the hERG channel with peptide toxin footprinting and molecular modeling. Biophys. J. 92, 3524-3540.

(17) Österberg, F., and Åqvist, J. (2005) Exploring blocker binding to a homology model of the open $\mathrm{hERG} \mathrm{K}^{+}$channel using docking and molecular dynamics methods. FEBS Lett. 579, 2939-2944.

(18) Farid, R., Day, T., Friesner, R. A., and Pearlstein, R. A. (2006) New insights about HERG blockade obtained from protein modeling, potential energy mapping, and docking studies. Bioorg. Med. Chem. $14,3160-3173$.

(19) Stary, A., Wacker, S. J., Boukharta, L., Zachariae, U., KarimiNejad, Y., Åqvist, J., Vriend, G., and de Groot, B. L. (2010) Toward a Consensus Model of the hERG Potassium Channel. ChemMedChem $5,455-467$.

(20) Ju, P. C., Pages, G., Riek, R. P., Chen, P. C., Chen, P. C., Torres, A. M., Bansal, P. S., Kuyucak, S., Kuchel, P. W., and Vandenberg, J. I. (2009) The Pore Domain Outer Helix Contributes to Both Activation and Inactivation of the hERG K ${ }^{+}$Channel. J. Biol. Chem. 284, 1000-1008.

(21) Pearlstein, R. A., Vaz, R. J., Kang, J. S., Chen, X. L., Preobrazhenskaya, M., Shchekotikhin, A. E., Korolev, A. M., Lysenkova, L. N., Miroshnikova, O. V., Hendrix, J., and Rampe, D. (2003) Characterization of HERG potassium channel inhibition using CoMSiA 3D QSAR and homology modeling approaches. Bioorg. Med. Chem. Lett. 13, 1829-1835.

(22) Zimbroff, D. L., Kane, J. M., Tamminga, C. A., Daniel, D. G., Mack, R. J., Wozniak, P. J., Sebree, T. B., Wallin, B. A., and Kashkin, K. B. (1997) Controlled, dose-response study of sertindole and haloperidol in the treatment of schizophrenia. Sertindole Study Group. Am. J. Psychiatry $154,782-791$.

(23) Rampe, D., Murawsky, M. K., Grau, J., and Lewis, E. W. (1998) The antipsychotic agent sertindole is a high affinity antagonist of the human cardiac potassium channel HERG. J. Pharmacol. Exp. Ther. 286, 788-793. 
(24) Lindström, E., Farde, L., Eberhard, J., and Haverkamp, W. (2005) QTc interval prolongation and antipsychotic drug treatments: Focus on sertindole. Int. J. Neuropsychopharmacol. 8, 615-629.

(25) http://www.fda.gov/downloads/AdvisoryCommittees/CommitteesMeetingMaterials/Drugs/PsychopharmacologicDrugsAdvisoryCommittee/UCM198218.pdf.

(26) Thomas, S. H. L., Drici, M. D., Hall, G. C., Crocq, M. A., Everitt, B., Lader, M. H., Le Jeunne, C., Naber, D., Priori, S., Sturkenboom, M., Thibaut, F., Peuskens, J., Mittoux, A., Tanghoj, P., Toumi, M., Moore, N. D., and Mann, R. D. (2010) Safety of sertindole versus risperidone in schizophrenia: Principal results of the sertindole cohort prospective study ( $\mathrm{SCoP})$. Acta Psychiatr. Scand. 122, 345-355.

(27) Åqvist, J., Medina, C., and Samuelsson, J. E. (1994) A new method for predicting binding affinity in computer-aided drug design. Protein Eng. 7, 385-391.

(28) Marelius, J., Kolmodin, K., Feierberg, I., and Aqvist, J. (1998) Q: A molecular dynamics program for free energy calculations and empirical valence bond simulations in biomolecular systems. J. Mol. Graphics Modell. 16, 213-225, 261.

(29) Marti-Renom, M. A., Stuart, A. C., Fiser, A., Sanchez, R., Melo, F., and Sali, A. (2000) Comparative protein structure modeling of genes and genomes. Annu. Rev. Biophys. Biomol. Struct. 29, 291-325.

(30) Lee, S. Y., Lee, A., Chen, J. Y., and MacKinnon, R. (2005) Structure of the KvAP voltage-dependent $\mathrm{K}^{+}$channel and its dependence on the lipid membrane. Proc. Natl. Acad. Sci. U.S.A. 102, $15441-15446$

(31) Jorgensen, W. L., Chandrasekhar, J., Madura, J. D., Impey, R. W., and Klein, M. L. (1983) Comparison of simple potential functions for simulating liquid water. J. Chem. Phys. 79, 926-935.

(32) Lindahl, E., Hess, B., and van der Spoel, D. (2001) GROMACS 3.0: A package for molecular simulation and trajectory analysis. J. Mol. Model. 7, 306-317.

(33) Jorgensen, W. L., Maxwell, D. S., and Tirado-Rives, J. (1996) Development and Testing of the OPLS All-Atom Force Field on Conformational Energetics and Properties of Organic Liquids. J. Am. Chem. Soc. 118, 11225-11236.

(34) Åqvist, J., and Luzhkov, V. (2000) Ion permeation mechanism of the potassium channel. Nature 404, 881-884.

(35) Jones, G., Willett, P., and Glen, R. C. (1995) Molecular Recognition of Receptor Sites using a Genetic Algorithm with a Description of Desolvation. J. Mol. Biol. 245, 43-53.

(36) Jones, G., Willett, P., Glen, R. C., Leach, A. R., and Taylor, R. (1997) Development and validation of a genetic algorithm for flexible docking. J. Mol. Biol. 267, 727-748.

(37) Eldridge, M. D., Murray, C. W., Auton, T. R., Paolini, G. V., and Mee, R. P. (1997) Empirical scoring functions. 1. The development of a fast empirical scoring function to estimate the binding affinity of ligands in receptor complexes. J. Comput.-Aided Mol. Des. 11, 425-445.

(38) Mooij, W. T. M., and Verdonk, M. L. (2005) General and Targeted Statistical Potentials for Protein-Ligand Interactions. Proteins 61, 272-287.

(39) Nervall, M., Hanspers, P., Carlsson, J., Boukharta, L., and Åqvist, J. (2008) Predicting binding modes from free energy calculations. J. Med. Chem. 51, 2657-2667.

(40) Luzhkov, V., and Åqvist, J. (2005) Ions and blockers in potassium channels: Insights from free energy simulations. Biochim. Biophys. Acta 1747, 109-120.

(41) Åqvist, J. (1996) Calculation of absolute binding free energies for charged ligands and effects of long-range electrostatic interactions. J. Comput. Chem. 17, 1587-1597.

(42) Luzhkov, V. B., Nilsson, J., Århem, P., and Åqvist, J. (2003) Computational modelling of the open-state $\mathrm{K}(\mathrm{v}) 1.5$ ion channel block by bupivacaine. Biochim. Biophys. Acta 1652, 35-51.

(43) Ryckaert, J. P., Ciccotti, G., and Berendsen, H. J. C. (1977) Numerical Integration of the Cartesian Equations of Motion of a System with Constraints: Molecular Dynamics of n-Alkanes. J. Comput. Phys. 23, 327-341.
(44) King, G., and Warshel, A. (1989) A surface constrained all-atom solvent model for effective simulations of polar solutions. J. Chem. Phys. 91, 3647-3661.

(45) Lee, F. S., and Warshel, A. (1992) A local reaction field method for fast evaluation of long-range electrostatic interactions in molecular simulations. J. Chem. Phys. 97, 3100-3107.

(46) Hansson, T., Marelius, J., and Åqvist, J. (1998) Ligand binding affinity prediction by linear interaction energy methods. J. Comput.Aided Mol. Des. 12, 27-35.

(47) Almlöf, M., Brandsdal, B. O., and Åqvist, J. (2004) Binding affinity prediction with different force fields: Examination of the linear interaction energy method. J. Comput. Chem. 25, 1242-1254.

(48) Marelius, J., Graffner-Nordberg, M., Hansson, T., Hallberg, A., and Åqvist, J. (1998) Computation of affinity and selectivity: Binding of 2,4-diaminopteridine and 2,4-diaminoquinazoline inhibitors to dihydrofolate reductases. J. Comput.-Aided Mol. Des. 12, 119-131.

(49) Ljungberg, K. B., Marelius, J., Musil, D., Svensson, P., Norden, B., and Åqvist, J. (2001) Computational modelling of inhibitor binding to human thrombin. Eur. J. Pharm. Sci. 12, 441-446.

(50) Ersmark, K., Feierberg, I., Bjelic, S., Hamelink, E., Hackett, F., Blackman, M. J., Hulten, J., Samuelsson, B., Åqvist, J., and Hallberg, A. (2004) Potent Inhibitors of the Plasmodium falciparum Enzymes Plasmepsin I and II Devoid of Cathepsin D Inhibitory Activity. J. Med. Chem. 47, 110-122.

(51) Andér, M., Luzhkov, V. B., and Åqvist, J. (2008) Ligand Binding to the Voltage-Gated Kv1.5 Potassium Channel in the Open State: Docking and Computer Simulations of a Homology Model. Biophys. J. 94, 820-831.

(52) Carlsson, J., Boukharta, L., and Åqvist, J. (2008) Combining Docking, Molecular Dynamics and the Linear Interaction Energy Method to Predict Binding Modes and Affinities for Non-nucleoside Inhibitors to HIV-1 Reverse Transcriptase. J. Med. Chem. 51, 2648-2656.

(53) Díaz, L., Bujons, J., Delgado, A., Gutiérrez-de-Terán, H., and Åqvist, J. (2011) Computational Prediction of Structure-Activity Relationships for the Binding of Aminocyclitols to $\beta$-Glucocerebrosidase. J. Chem. Inf. Model. 51, 601-611.

(54) Crowley, P. J., Robinson, M. J. T., and Ward, M. G. (1977) Conformational effects in compounds with 6-membered rings. XII. The conformational equilibrium in $\mathrm{N}$-methylpiperidine. Tetrahedron 33, 915-925.

(55) Wold, S., Esbensen, K., and Geladi, P. (1987) Principal Component Analysis. Chemom. Intell. Lab. Syst. 2, 37-52.

(56) Mitcheson, J. S., Chen, J., Lin, M., Culberson, C., and Sanguinetti, M. C. (2000) A structural basis for drug-induced long QT syndrome. Proc. Natl. Acad. Sci. U.S.A. 97, 12329-12333.

(57) Perry, M., de Groot, M. J., Helliwell, R., Leishman, D., TristaniFirouzi, M., Sanguinetti, M. C., and Mitcheson, J. (2004) Structural determinants of HERG channel block by clofilium and ibutilide. Mol. Pharmacol. 66, 240-249.

(58) Kamiya, K., Niwa, R., Mitcheson, J. S., and Sanguinetti, M. C. (2006) Molecular determinants of hERG channel block. Mol. Pharmacol. 69, 1709-1716.

(59) Perry, M., Stansfeld, P. J., Leaney, J., Wood, C., de Groot, M. J., Leishman, D., Sutcliffe, M. J., and Mitcheson, J. S. (2006) Drug binding interactions in the inner cavity of hERG channels: Molecular insights from structure-activity relationships of clofilium and ibutilide analogs. Mol. Pharmacol. 66, 509-519.

(60) Siebrands, C. C., and Friederich, P. (2007) Structural requirements of human ether-a-go-go-related gene channels for block by bupivacaine. Anesthesiology 106, 523-531.

(61) Schwoerer, A. P., Blutner, C., Brandt, S., Binder, S., Siebrands, C. C., Ehmke, H., and Friederich, P. (2007) Molecular interaction of droperidol with human ether-a-go-go-related gene channels: Prolongation of action potential duration without inducing early afterdepolarization. Anesthesiology 106, 967-976.

(62) Kamiya, K., Niwa, R, Morishima, M., Honjo, H., and Sanguinetti, M. C. (2008) Molecular Determinants of hERG Channel Block by Terfenadine and Cisapride. J. Pharmacol. Sci. 108, 301-307. 
(63) Kamiya, K., Mitcheson, J. S., Yasui, K., Kodarna, I., and Sanguinetti, M. C. (2001) Open channel block of HERG K ${ }^{+}$channels by vesnarinone. Mol. Pharmacol. 60, 244-253.

(64) Fernandez, D., Ghanta, A., Kauffman, G. W., and Sanguinetti, M. C. (2004) Physicochemical features of the hERG channel drug binding site. J. Biol. Chem. 279, 10120-10127.

(65) Witchel, H. J., Dempsey, C. E., Sessions, R. B., Perry, M., Milnes, J. T., Hancox, J. C., and Mitcheson, J. S. (2004) The lowpotency, voltage-dependent HERG blocker propafenone: Molecular determinants and drug trapping. Mol. Pharmacol. 66, 1201-1212.

(66) Hosaka, Y., Iwata, M., Kamiya, N., Yamada, M., Kinoshita, K., Fukunishi, Y., Tsujimae, K., Hibino, H., Aiziwa, Y., Inanobe, A., Nakamura, H., and Kurachi, Y. (2007) Mutational Analysis of Block and Facilitation of HERG Current by A Class III Anti-Arrhythmic Agent, Nifekalant. Channels 1, 198-208. 